\title{
Les défis de l'optique adaptative en astronomie
}

\author{
Gérard ROUSSET, Damien GRATADOUR et Eric GENDRON \\ LESIA, Université Paris Diderot et Observatoire de Paris \\ gerard.rousset@obspm.fr
}

Depuis son introduction en astronomie il y a une vingtaine d'années, l'optique adaptative a permis des avancées spectaculaires en termes de qualité d'image et a conduit à des découvertes majeures dans le domaine de l'astrophysique : cartographie du sol de Titan, confirmation de la présence d'un trou noir massif au centre de la Voie lactée, étude de sources extra-galactiques...

Les développements récents visent d'une part à augmenter la qualité de la correction afin d'imager des exoplanètes, et d'autre part à étendre le champ corrigeable. De nouvelles techniques d'optique adaptative, comme l'optique adaptative multi-objets ou l'optique adaptative multi-conjuguée, ont ainsi été développées ouvrant la voie à de nouvelles observations et à la construction de télescopes extrêmement grands, avec un diamètre de 30 mètres ou plus.

\section{Quelques rappels}

Les grands télescopes astronomiques au sol sont affectés par la turbulence de l'atmosphère terrestre qui limite à environ 1" (seconde d'arc) leur pouvoir de résolution angulaire. L'optique adaptative $(\bigcirc \mathrm{A})$ permet de corriger en temps réel ces perturbations turbulentes et ainsi de recouvrer la limite de résolution $\lambda / D$ de ces télescopes, imposée par la diffraction où $\lambda$ est la longueur d'onde d'observation et $D$ leur diamètre (voir Photoniques n³3). Cette limite vaut $0,04^{\prime \prime}$ à 1, 6 m pour un diamètre de 8 mètres. Le principe de l'OA repose sur la déformation en temps réel, par quelques centaines d'actionneurs pour l'exemple précédent, d'un miroir mince placé dans le faisceau optique en sortie du télescope, qui compense les fluctuations spatiales et temporelles en différence de marche afin de restituer un front $d$ 'onde quasiment plan. Les défauts de planéité de l'onde sont mesurés en quelques centaines de points par un analyseur de front d'onde (AFO) dont les signaux sont traités par un calculateur temps réel pour permettre la commande du miroir déformable (MD). La fréquence d'échantillonnage de cette boucle d'asservissement est généralement de quelques centaines de Hertz.
Les principes de l'OA ont été proposés par un astronome américain, H. Babcock, en 1953. Cependant, il a fallu attendre le milieu des années 70 pour les premières démonstrations classifiées dans le domaine de la Défense, notamment aux États-Unis et en France. Ce n'est pourtant qu'en 1989 sous l'impulsion de Pierre Léna qu'une équipe française issue de I'Observatoire de Paris, de I'ONERA et de la CGE (aujourd'hui CILAS) associé à I'European Southern Observatory (ESO) en démontre pour la première fois toutes les potentialités pour l'astronomie avec le prototype COME-ON (21 actionneurs) dans le cadre de la préparation du Very Large Telescope (VLT) [1]. Ces laboratoires français en pointe dans leur domaine ont ensuite développé plusieurs systèmes d'OA : les versions améliorées du premier prototype (COME-ON+ et ADONIS) installées au télescope de 3,6 mètres (ESO) de La Silla au Chili, le système PUEO au Télescope Canada France Hawaï, puis en 2001 le premier système d'OA du VLT appelé NAOS ( 190 actionneurs). Un exemple d'image produite par $\bigcirc A$ est donné en figure 1 en comparaison de l'image sans correction. On constate que la haute résolution a été recouvrée lle premier anneau d'Airy est même observable) ce qui permet de séparer un grand nombre d'objets dans cet amas ; en outre la sensibilité a été accrue car de nombreuses étoiles très faibles sont maintenant détectables du fait de la concentration des photons collectés dans le pic de la diffraction.

Mais I'OA peut souffrir de deux limitations importantes dues à la luminosité limitée des sources astronomiques et aux effets d'anisoplanétisme [1]. En effet, I'AFO doit détecter une certaine quantité de photons pour effectuer correctement la mesure. Il faut donc disposer d'une source suffisamment brillante, dite étoile guide. Souvent l'objet observé est trop faiblement lumineux, il faut alors chercher une étoile brillante environnante mais hélas à proximité immédiate à cause de l'anisoplanétisme, réduisant sérieusement la couverture du ciel. L'anisoplanétisme est induit par la décorrélation angulaire des fronts d'onde issus de différentes directions d'observation du fait de la présence de couches assez fortement turbulentes en altitude, généralement au niveau de la tropopause (à environ $10 \mathrm{~km}$ ). Le champ de vue corrigeable par $\bigcirc A$ autour d'une étoile guide est typiquement de quelques dizaines de secondes d'arc dans le proche infrarouge. Les astronomes ont 

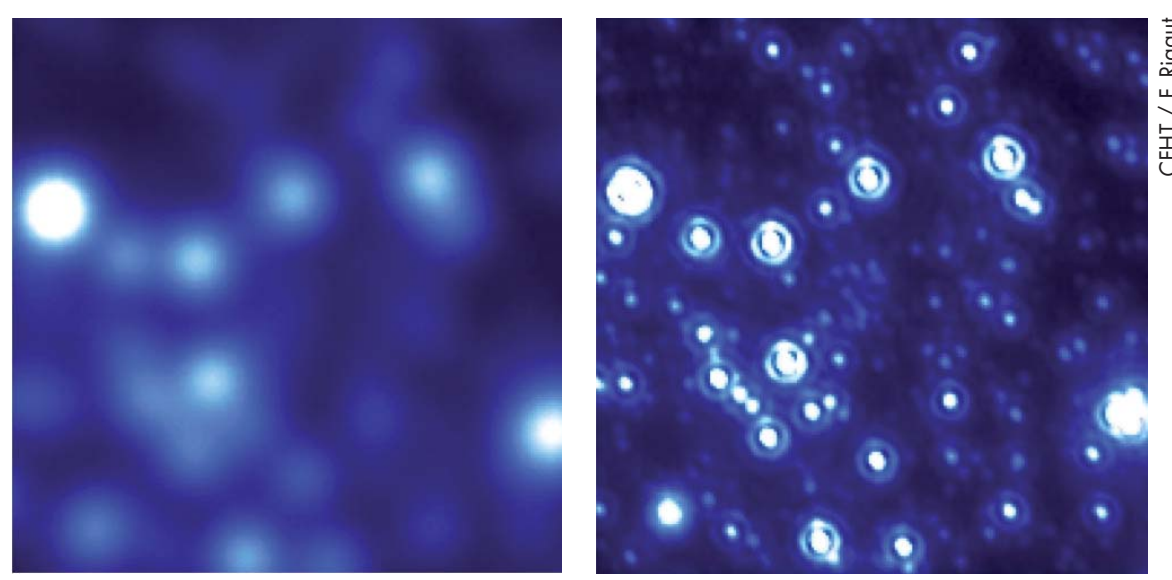

Figure 1. Images à 2,2 $\mu \mathrm{m}$ (bande K) de l'amas d'étoiles autour du centre galactique au télescope Canada France Hawaï. À gauche, image sans correction. À droite, image corrigée par le système d'OA PUEO.

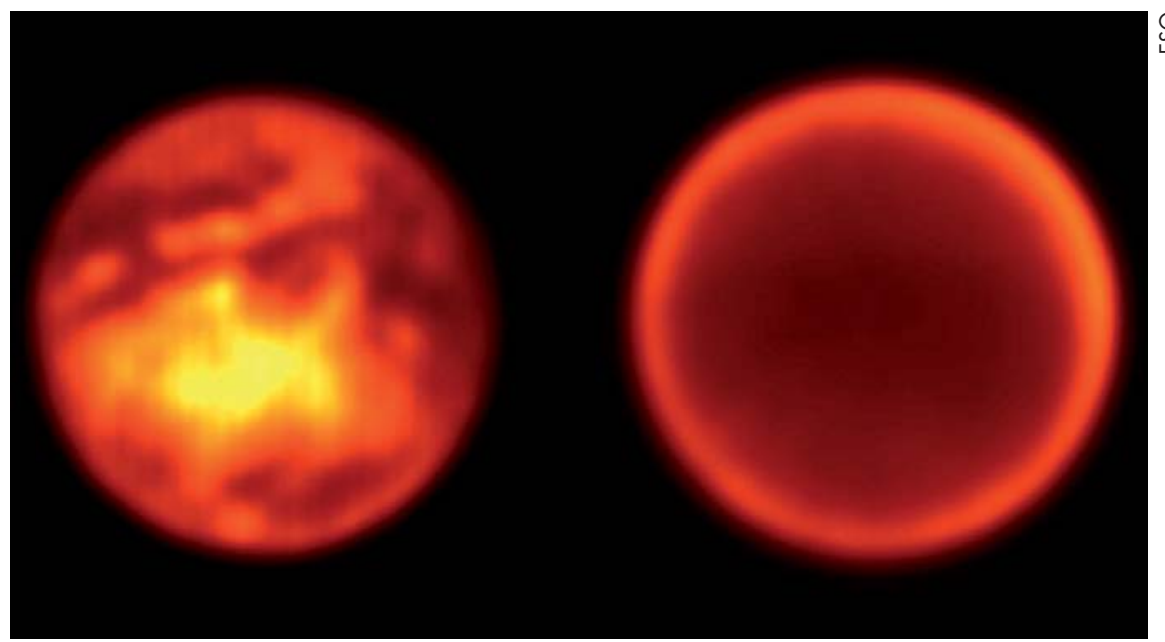

Figure 2. Images de Titan obtenues simultanément grâce au mode d'imagerie différentielle de NACO. À gauche : à 1,575 $\mu \mathrm{m}$ une fenêtre de transparence du méthane dévoilant la surface et à droite à $1,625 \mu \mathrm{m}$, une vue de l'atmosphère.

donc imaginé une solution en créant artificiellement une étoile dans I'atmosphère par un tir laser, dite étoile laser. En général, le laser est continu et accordé sur la raie D2 du sodium pour créer une source lumineuse par les atomes de sodium résonnants présents à environ $90 \mathrm{~km}$ d'altitude dans la mésosphère. Cette étoile laser présente cependant des limitations en performance : elle ne permet de mesurer ni le basculement ni la défocalisation du front d'onde et elle conduit à une forme d'anisoplanétisme, dite effet de cône, du fait qu'elle est à distance finie alors que les étoiles naturelles sont à l'infini. Malgré tout, on peut montrer que l'utilisation de l'étoile laser permet d'accroître quelque peu la couverture du ciel. Mais l'étape ultime serait bien sûr de pouvoir s'affranchir de l'anisoplanétisme par des AO " grand champ» (voir paragraphe 3).

\section{Quelques résultats}

Les systèmes d' $\bigcirc A$, introduits ci-dessus, ont été utilisés de manière routinière pour mener à bien de nombreux programmes astrophysiques et ont permis des découvertes importantes. Nous les illustrons ici par quelques résultats obtenus avec l'instrument NACO installé sur le VLT, associant NAOS et CONICA, caméra pour l'imagerie infrarouge.
Dans le système solaire, NACO a permis d'obtenir des images d'une finesse inégalée de certains corps comme lo, un satellite de Jupiter au volcanisme très actif, ou Titan, un satellite de Saturne très particulier qui possède une croûte solide et une atmosphère riche et dense saturée en méthane (figure 2). Ces images ont permis non seulement d'établir la cartographie complète du sol de Titan, mais aussi d'étudier précisément la météorologie de ce satellite, en complément des missions spatiales in situ.

NACO a aussi permis la découverte d'une planète au cœur du disque de débris de la jeune étoile Beta Pictoris à environ 60 années lumières du Soleil (voir article de Daniel Rouan dans ce numéro). Le rayon de son orbite est comparable à celui de l'orbite de Saturne.

L'arrivée du VLT équipé d'OA a permis de franchir un pas décisif dans l'étude de notre galaxie. Grâce à la finesse des images obtenues avec cet instrument, la présence d'un trou noir super-massif au centre de la Voie lactée a été confirmée. Pour la première fois, la position et les caractéristiques de ce trou noir, dont on soupçonnait l'existence depuis de nombreuses années, ont pu être déterminées avec précision grâce à l'étude de la trajectoire des étoiles orbitant autour de cet objet très compact et invisible. L'instrument a aussi permis de mettre en évidence des sursauts infrarouge dans son activité, probablement causés par de la matière tombant sur celui-ci (figure 3).

La haute résolution et la sensibilité offertes par NACO, ainsi que les multiples techniques instrumentales qui peuvent lui être associées, ont aussi été mises à profit pour l'étude de sources extra-galactiques comme Messier 77, une galaxie située à environ 45 millions d'annéeslumière. Grâce à des observations dans le proche infrarouge, des structures spatiales très particulières en forme de vagues ont été mises en évidence au coeur de cette galaxie dite à noyau actif et les mesures spectroscopiques effectuées sur celles-ci ont révélé la présence d'amas d'étoiles géants en leur cœur, enfouis sous d'énormes quantités de gaz et de poussière (figure 4). 


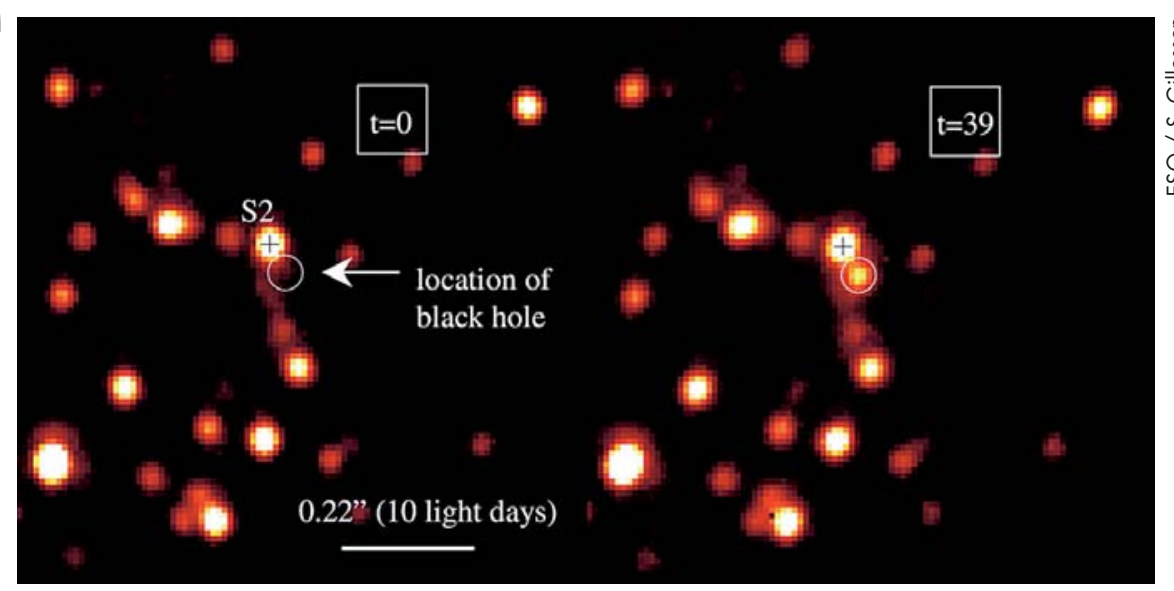

Figure 3. Images à 2,2 $\mu \mathrm{m}$ (bande K) de la zone centrale du Centre Galactique montrant la position du trou noir à gauche et à droite un exemple de sursaut d'activité.

\section{Challenges actuels}

Deux axes cruciaux de développement sont actuellement poursuivis en astronomie : l'augmentation de la qualité de correction dans le but d'imager des planètes extrasolaires etl'accroissement du champ de vue corrigeable par l'OA [2]

La détection directe des exoplanètes permettra de caractériser leurs propriétés physico-chimiques. Pour cela, il faut être capable de distinguer leurs photons du halo de lumière dominant dû à l'étoile hôte et donc d'obtenir à la fois une haute résolution angulaire, pour séparer par exemple la distance Terre - Soleil vue à 33 années lumière $\left(0,1^{\prime \prime}\right)$, et un très haut contraste dans les images pour détecter des rapports de luminosité étoile-compagnon jusqu' à $10^{6}$ pour des géantes ga- zeuses. Pour les très grands télescopes au sol, I'OA dite extrême (XAO) est développée dans le but d'atteindre de telles performances. L'écart quadratique moyen spatial du front d'onde, de l'ordre de $3 \mu \mathrm{m}$ pour la turbulence atmosphérique, doit être ramené à moins de $80 \mathrm{~nm}$ pour la bande $\mathrm{H}$ de l'atmosphère $(1,65 \mu \mathrm{m})$, toutes aberrations confondues. Cependant, cette qualité de l'onde ne suffit pas, il faut associer à la XAO la coronographie : pour sortir le signal de l'exoplanète, la lumière de l'étoile est rejetée par un masque plan focal et un diaphragme pupillaire selon le principe inventé par Bernard Lyot. Enfin, les techniques d'imagerie différentielle sont en plus nécessaires pour distinguer l'exoplanète parmi les tavelures stellaires dues aux aberrations résiduelles. Un instru- ment de ce type, nommé SPHERE ( 1400 actionneurs, voir Photoniques $n^{\circ} 33$ l, est actuellement en phase d'intégration en France avant d'être installé sur le VLT à la fin de 2012. Il est développé par un consortium européen mené par l'Institut de planétologie et d'astrophysique de Grenoble (IPAG). D'autres projets de ce type existent aussi aux États-Unis, comme le GEMINI Planet Imager.

Aujourd'hui, les astronomes développent de nouveaux systèmes d'OA offrant à la fois la couverture du ciel par l'utilisation d'étoiles laser et un grand champ de vue par des approches de sondage et correction volumique de l'atmosphère (encadré 1 et figure 5). L'idée consiste à tirer plusieurs lasers dans l'atmosphère dans des directions suffisamment différentes pour sonder, sur le trajet retour des photons, tout le volume de l'atmosphère correspondant au champ de vue d'intérêt à corriger. La correction se fait en plaçant plusieurs miroirs déformables conjugués à différentes altitudes dans l'atmosphère c'est l'OA multi-conjuguée (MCAO). Ce principe a été démontré une première fois par I'ESO au VLT en utilisant des étoiles guides naturelles. Mais c'est avec des étoiles laser que ce concept prend tout son intérêt. Un tel système vient d'obtenir sa première lumière sur le télescope américain GEMINI de 8 mètres au Chili et en démontre la puissance (figure 6). II est équipé de trois MD répartis en altitude et de 5 étoiles laser complétées par trois étoiles guides naturelles recherchées dans un champ de 3' (minutes d'arc).

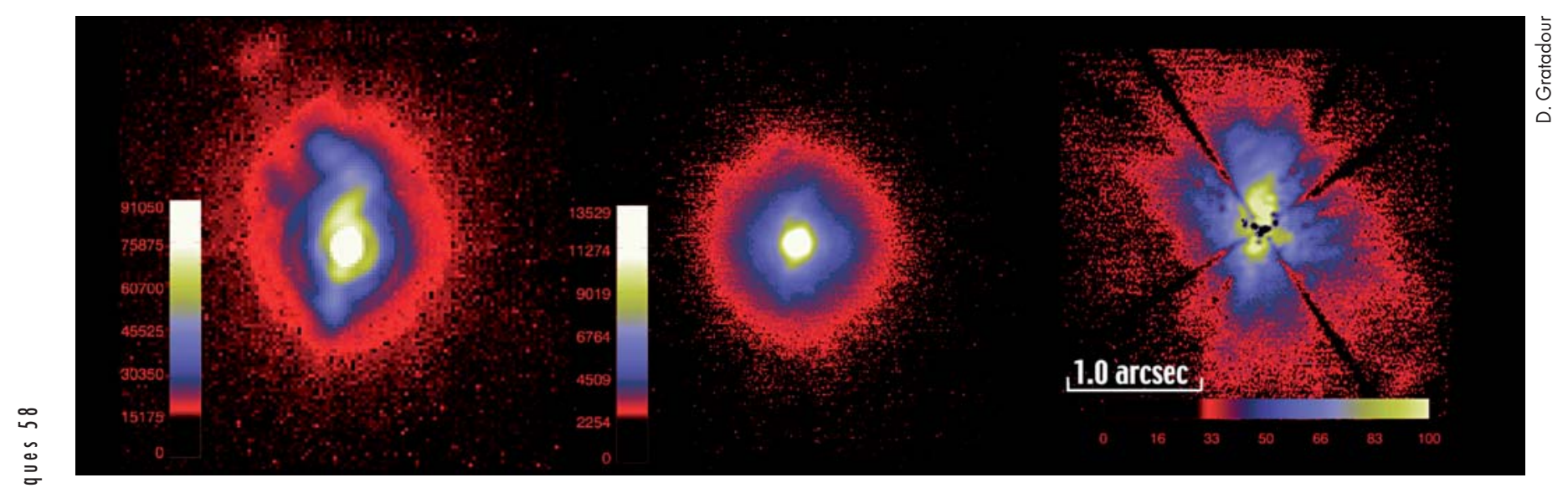

Figure 4. Images du noyau actif de Messier 77. À gauche : en bande Mà $4 \mu \mathrm{m}$. Au centre : en bande Ks à 2,2 $\mu \mathrm{m}$. À droite : en bande Ks mais avec un coronographe, celui-ci permet de mettre en évidence des structures à fort contraste au Nord-Est de la source centrale. 

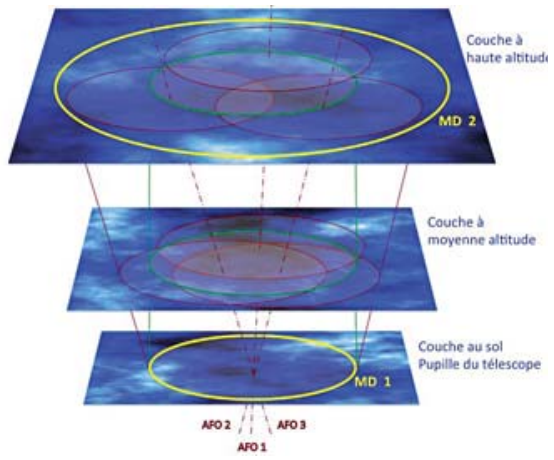

Figure 5. Schéma d'une configuration typique d'observation pour l'OA grand champ, ici l'OA multi-conjuguée. Les faisceaux de trois étoiles guides (en rouge) traversentl'atmosphère formée de trois couches turbulentes et alimente trois $\mathrm{AFO}$. À partir de ces mesures, le volume de turbulence est reconstruit par tomographie et la correction à appliquer à tout le champ de vue est obtenue par projection du volume sur les deux MD, I'un dans la pupille, conjugué de la couche au sol, l'autre conjugué à haute altitude. Le faisceau dans la direction d'observation (vert) est ainsi corrigé par les deux MD.

L'ESO développe de son côté un système versatile de correction de la seule couche au sol (GLAO) très grand champ $\left(7,5^{\prime}\right)$ pour le proche infrarouge et de tomographie laser (LTAO) petit champ pour le visible.

Mais si l'on veut combiner une assez bonne qualité de correction et un très grand champ de vue, on ne peut plus envisager un système MCAO du fait des contraintes imposées par la conservation de l'étendue de faisceau. Il faut alors diviser le champ! C'est possible quand on souhaite observer en parallèle plusieurs galaxies à très grand redshift dans un champ cosmologique de l'ordre de 10'. II faut utiliser une $\bigcirc A$ répartie, dite multiobjet (MOAO). Dans ce cas, les AFO mesurent le volume turbulent sur des étoiles guides lasers et naturelles dans des directions du ciel différentes de celles des objets observés. Les MD, eux, sont installés dans chacun des trains optiques de chaque objet observé. Les AFO sont aveugles aux résidus de correction des $M D$ : le système fonctionne donc en boucle ouverte. Une équipe franco-anglaise menée par l'Observatoire de Paris vient récemment de tester
Optique adaptative grand champ

Pour pouvoir corriger un grand champ de vue, il faut être capable de synthétiser le volume turbulent correspondant, présent au-dessus du télescope. Pour sonder ce volume, on choisit plusieurs directions de mesure du front $d^{\prime}$ onde sur des étoiles guides, le plus souvent des étoiles laser : sur l'exemple de la figure 5, sont utilisés trois AFO pour trois étoiles guides. Le volume des perturbations turbulentes peut être alors reconstruit dans le calculateur temps réel par des algorithmes de tomographie utilisant un modèle d'atmosphère turbulente. La correction du volume est effectuée par plusieurs MD conjugués sur les couches prépondérantes de l'atmosphère à différentes altitudes. Les commandes sont obtenues par projection sur les MD des perturbations reconstruites. Sur la figure 5, seuls deux MD sont utilisés ; les couches intermédiaires ne sont donc que partiellement corrigeables. Généralement, on place au moins un $M D$ conjugué de la couche au sol, c'est-à-dire dans la pupille d'entrée. Là, la correction est commune à toutes les directions d'observation. En altitude par contre, le MD doit couvrir l'ensemble des empreintes des faisceaux pour tous les objets du champ d'intérêt et donc chaque zone de ce MD ne corrige plus que certaines directions.

À partir de ce principe de base, dit de l'OA multi-conjuguée (Multi Conjugate Adaptive Optics, MCAO), plusieurs concepts d'OA sont aujourd'hui à l'étude. Un concept utilise la tomographie pour compenser l'effet de cône des étoiles laser afin d'obtenir une très bonne qualité de correction sur l'axe (Laser Tomography Adaptive Optics, LTAO). Un autre consiste à ne corriger que la couche au sol (Ground Layer Adaptive Optics, GLAO) par un MD dans la pupille, donnant accès à un très grand champ pour une très faible qualité de correction. Enfin un dernier concept vise à ne corriger que quelques directions bien particulières et pas tout le champ, en utilisant un MD par objet, conjugué de la pupille dans un train optique dédié et commandé en boucle ouverte. On parle d'OA multi-objet (Multi Object Adaptive Optics, MOAO). La mesure du volume se fait toujours sur plusieurs étoiles guides mais ici indépendantes des objets et donc sans rebouclage sur les MD. La correction pour chaque objet peut être d'assez bonne qualité dans un champ très grand. Dans tous ces systèmes, on doit combiner les mesures sur étoiles laser et étoiles naturelles pour assurer les meilleures correction et couverture du ciel possibles.

\section{QOPTIE}

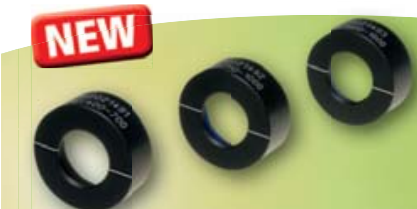

Achromats UV-VIS

- UV-VIS 240-700 nm

- Compatible avec le système de cage Microbench
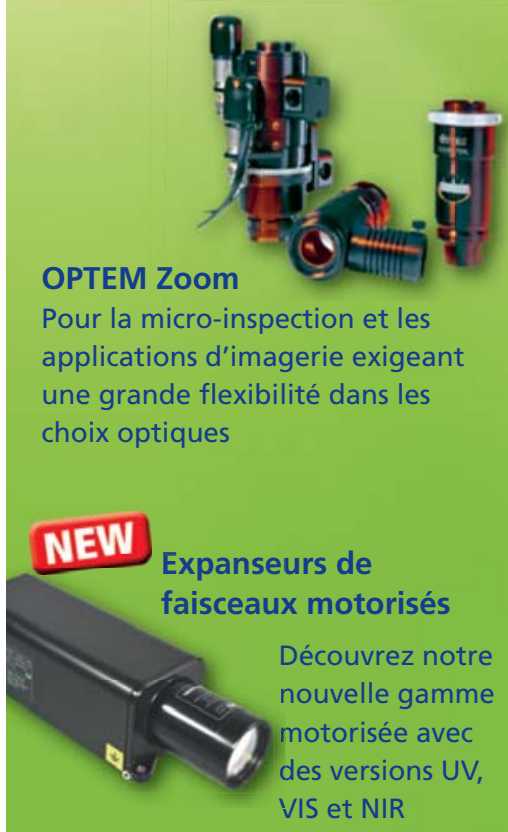

Qioptiq Photonics France info@qioptiq.fr

Tel: +33(0)472520420

Fax: +33 (0)472539296

www.qioptiq.fr

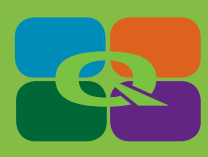




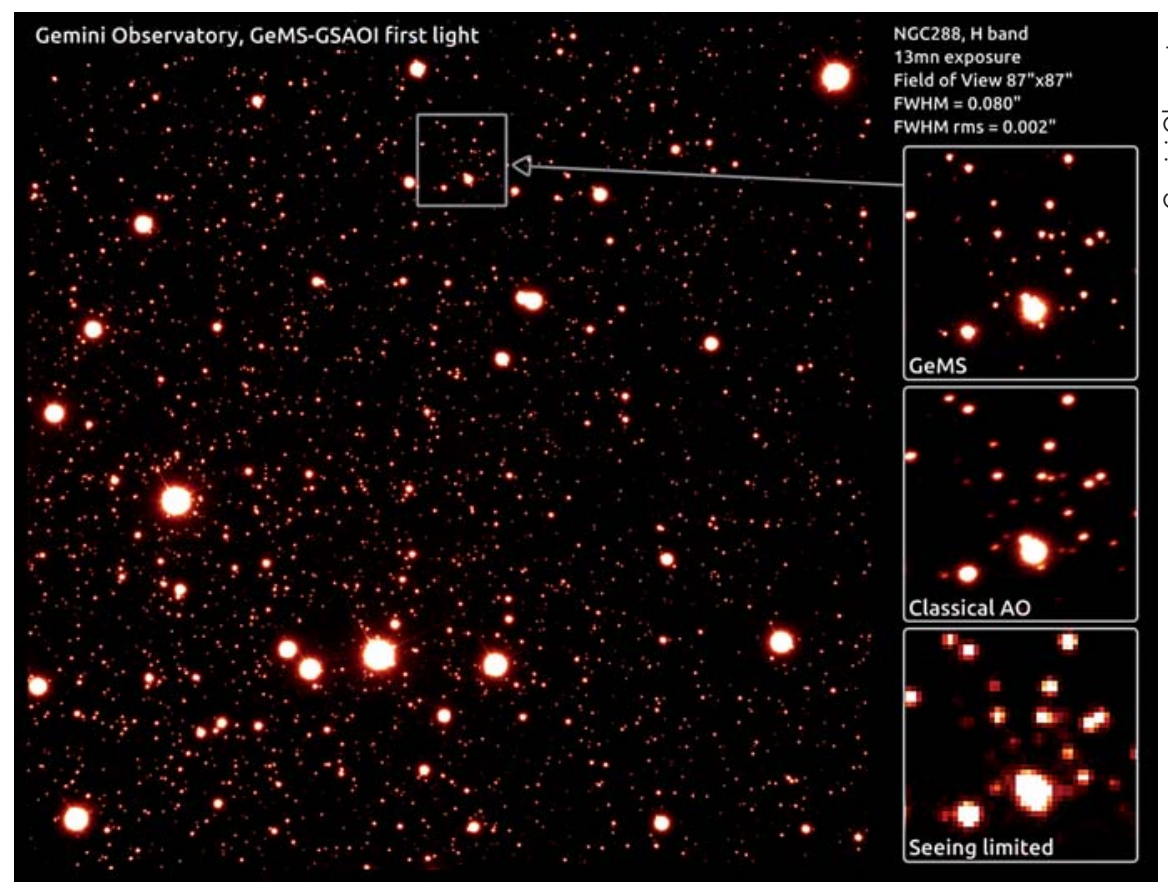

Figure 6. Image du champ stellaire NGC288 corrigé par MCAO au télescope GEMINI sud (GeMS), champ de vue de $87^{\prime \prime}$, bande $\mathrm{H}(1,65 \mu \mathrm{m})$, largeur à mi-hauteur obtenue des images d'étoiles de l'ordre de $0,08^{\prime \prime}$ très homogène dans le champ.

et démontrer la faisabilité de ce concept sur le ciel avec des étoiles guides naturelles (figure 7). La prochaine étape est bien sûr de le faire avec des étoiles laser.

\section{Perspectives}

Mais les ambitions des astronomes ne s'arrêtent pas là. En effet, cette décennie devrait voir le lancement de projets de télescopes extrêmement grands, de 30 à 40 mètres de diamètre, afin de pouvoir par exemple sonder les profondeurs de l'univers jusqu' à l'époque de la formation des premières étoiles et galaxies et, plus proche de nous, de caractériser la diversité des systèmes planétaires extrasolaires (voir Photoniques $n^{\circ} 48$ ). Ces télescopes devront être équipés d'OA pour rendre accessibles les performances en résolution angulaire et sensibilité liées à leur diamètre gigantesque. II s'agit suivant les cas de LTAO, MCAO ou MOAO. Le nombre d'actionneurs requis sur les MD et le nombre de points de mesure des AFO atteint plusieurs milliers, conduisant à des puissances de calcul temps réel encore difficiles à mettre en œuvre aujourd'hui. Par ailleurs, dans chaque cas, I'utilisation des étoiles laser devra permettre d'atteindre les qualités de correction requises dans le champ et d'assurer une couverture de ciel maximale. Mais avec de tels diamètres, de nouveaux problèmes apparaissent. Les AFO résolvent en profondeur la couche de $\mathrm{Na}$ mésosphérique impliquant une dépendance de la mesure aux variations de densité et de distribution des atomes. De nouvelles techniques de mesure sont à développer pour maîtriser ou compenser ces effets.

Le nombre de défis à relever est très important pour ces futurs projets et les équipes françaises y travaillent d'arrachepied. Elles sont souvent à la pointe de l'innovation dans ce domaine.

\section{Références}

[1] F. Roddier (Ed.), Adaptive Optics in Astronomy. Cambridge University Press (1999).

[2] Yann Clénet, Jean-Marc Conan, Thierry Fusco, Gérard Rousset (Eds.), Adaptive Optics for Extremely Large Telescopes, 22-26 June 2009, Paris, Proceedings. EDP Sciences (2010). http://ao4elt.edpsciences.org/

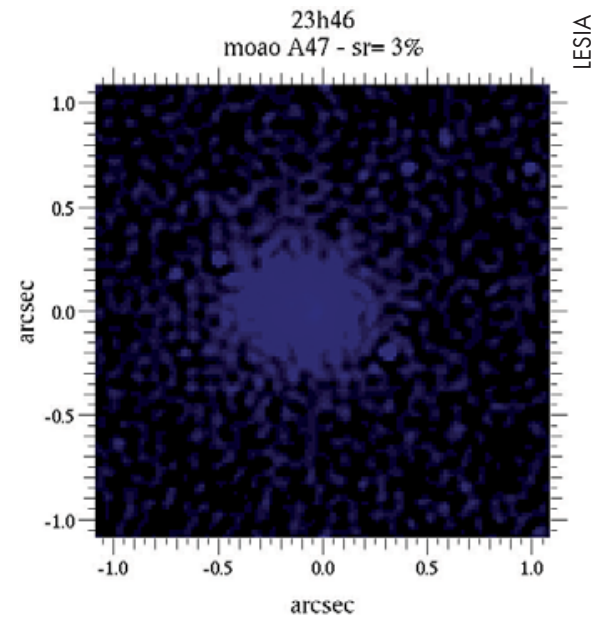

$00 \mathrm{~h} 27 \mathrm{~m} 42$

moao $\mathrm{A} 47$ - $\mathrm{sr}=25 \%$

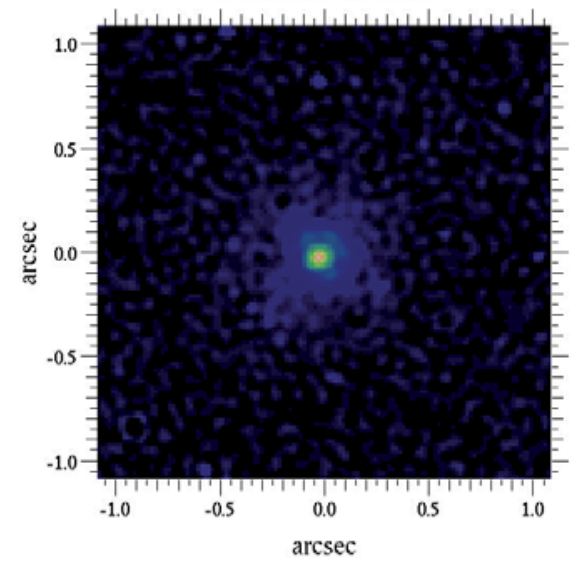

Asterism \#47

Coordonnees : $21 \mathrm{~h} 12 \mathrm{m01s} 38^{*} 35^{\prime} 56^{\prime \prime}$ Observable de $18 \mathrm{~h} 53 \mathrm{~m} 24 \mathrm{~s}$ a $02 \mathrm{h03m03s}$ TU

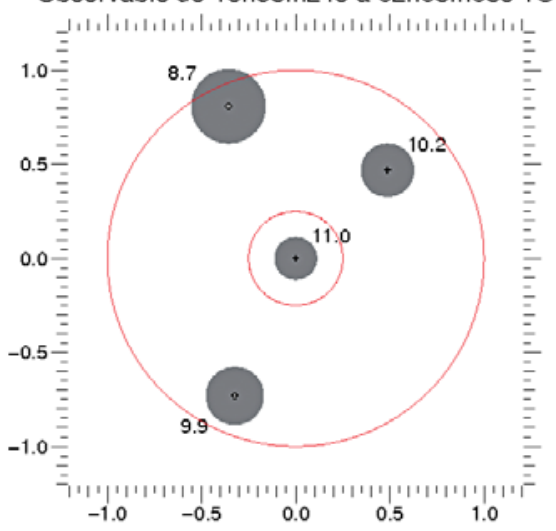

Figure 7. Images d'une étoile obtenues au télescope W. Herschell de 4,2 mètres (Iles Canaries). En haut, non corrigée. Au centre, corrigée par le système d'OA multi-objets (MOAO) CANARY utilisant un MD en boucle ouverte placé dans le train optique de l'étoile imagée sur l'axe et trois AFO en boucle ouverte mesurant le volume turbulent sur trois étoiles guides naturelles hors axe. En bas: configuration de l'astérisme d'étoiles naturelles utilisées, le grand cercle rouge est de l' de rayon. 